\title{
Smokeless tobacco products profile and pictorial warning labels in India: A review
}

\author{
Fareeda B. Shaik ${ }^{1}$, Narendra Maddu ${ }^{1}$
}

\section{AFFILIATION}

1 Department of Biochemistry, Sri Krishnadevaraya University, Anantapur, India

\section{CORRESPONDENCE TO}

Narendra Maddu. Department of Biochemistry, Sri Krishnadevaraya University, Anantapur, 515003, Andhra Pradesh, India. E-mail: dr.narendramaddu@gmail.com

\section{KEYWORDS}

smokeless tobacco products, pictorial health warning labels, panmasala, gutkha, khaini

Received: 8 January 2019, Revised: 27 March 2019,

Accepted: 29 November 2019

https://doi.org/10.18332/popmed/114940

\begin{abstract}
INTRODUCTION Smokeless tobacco (SLT) companies produce different SLT brands and types that are used extensively throughout India.

METHODS Within this cross-sectional pack-collection study, available information on details, composition, and profiles of various SLT products and the pictorial warning labels were collected from available retail paan shops in Ananthapur town, Andhra Pradesh, India.

RESULTS SLT products are defined by specified names according to regional levels and the products of SLT have their own product constituents. The smokeless tobacco products like madhu chhap, hans chhap, and miraj were identified. The gutkha brands are in the form of khaleja and
\end{abstract}

rebel, and are available in the Indian tobacco market. The brands of vimal, hira, rajnigandha, and RMD are panmasala products in the Ananthapur region. The health warning labels are present on the packets of most of the SLT products in the form of 'Tobacco kills/Tobacco causes mouth cancer/ Chewing of tobacco is injurious to health/Chewing of panmasala may be injurious to health'.

CONCLUSIONS This limited pack-collection study identified a plethora of SLT products on the Indian market with an array of ingredients and warning labels - including graphic warning labels. These findings suggest that the monitoring of pictorial health warnings on tobacco product packaging as prevention efforts/measures is warranted.

\section{INTRODUCTION}

Tobacco consumption is a major contributing and risk factor and the 6th leading cause of death globally ${ }^{1}$. Smokeless tobacco is consumed in an unburnt form and has been widely used orally or through nasal inhalation ${ }^{2}$. Smokeless tobacco products have differences in their composition, preparation, and toxicity, but they all consisting of a nicotine alkaloid $^{3}$. The chemical constituents or main ingredients present in the smokeless tobacco products are nicotine, tobacco-specific N-nitrosamines (TSNA), nitrosamine acids, polycyclic aromatic hydrocarbons (PAHs), aldehydes, and heavy metals ${ }^{4}$. Globally, the consumption of smokeless tobacco is largely concentrated in India and Bangladesh. These two countries have $80 \%$ of the 300 million smokelesstobacco users worldwide ${ }^{5,6}$. The tobacco industry invests half a billion dollars annually to promote the manufacture of SLT products, twice as much as earlier ${ }^{7,8}$. Recently, the Food and Drug Administration (FDA) proposed a rule to reduce the amount of tobacco-specific N-nitrosamines in SLT products in the United States due to their carcinogenic activity ${ }^{9}$.

Smokeless tobacco users who dip or chew 8-10 times a day might ingest a similar amount of nicotine as cigarette smokers who smoke 30-40 cigarettes per day ${ }^{10,11}$. The consumption of smokeless tobacco is higher due to the greater social acceptance, curiosity, and culture, which are influencing factors for SLT initiation and prevalence. About $35-40 \%$ of tobacco consumption in India is in smokeless tobacco forms, most of which is Nicotiana rustica, while most smoking tobacco is Nicotiana tabacum ${ }^{12}$. There are several types of chewing habits like the use of betel quid, khaini, mawa, panmasala plain without tobacco, and gutkha ${ }^{13}$. Promotion of SLT use has increased as non-users start to use SLT, which serves as an alternative to smoking and leads to dual-users of tobacco ${ }^{14,15}$ with the number of consumers increasing annually.

To combat the above epidemic, the Indian government has introduced warning labels for cigarettes or other tobacco 
products ${ }^{16}$. Tobacco warning labels are a useful tobacco control tool, however research has shown that attention to warnings and exposure to current health warning labels is lowest among individuals with lower education and income ${ }^{17,18}$. Health warnings on product packaging are a costeffective, population-wide strategy to inform populations about the health risks associated with tobacco consumption ${ }^{19}$.

The aim of the present study is to examine the reported composition, characteristics and manufacturers of smokeless tobacco products in India.

\section{METHODS}

This was a cross-sectional pack-collection study. Available information on details and product profiles of various SLT products and pictorial warnings labels were collected from retail shops in Ananthapur, India. The different SLT products include panmasala, gutkha, khaini and tobacco brands, purchased from nearby paan shops. The baba chewing tobacco is available in various types and this information was collected from owners of paan shops.

\section{RESULTS}

Tables 1-3 depict the current types of smokeless tobacco products available for sale in Ananthapur, India, including their reported ingredients, price, and manufacturer.

Figures 1 and 2 present the images of different smokeless

Table 1. Tobacco brands available in Andhra Pradesh, India

\begin{tabular}{|c|c|c|c|c|c|}
\hline No & Tobacco brands & Reported ingredients & $\begin{array}{l}\text { Net weight } \\
\text { (g) }\end{array}$ & Tobacco company & $\begin{array}{l}\text { Price } \\
\text { (Rs) }\end{array}$ \\
\hline 1 & Swagat gold & $\begin{array}{l}\text { Unmanufactured raw tobacco } \\
\text { Not a gutkha or panmasala }\end{array}$ & 25 & $\begin{array}{l}\text { Qutab manufacturing company, } \\
\text { Bakoli, Delhi }\end{array}$ & $20 /-$ \\
\hline 2 & Raja & $\begin{array}{l}\text { Tobacco spit product } \\
\text { Not a gutkha/ panmasala/ supari }\end{array}$ & 6 & $\begin{array}{l}\text { Lokenath Prasad Gupta, Patparganj, } \\
\text { Delhi }\end{array}$ & $5 /-$ \\
\hline 3 & Madhu chhap & $\begin{array}{l}\text { Manufactured tobacco } \\
\text { Not a gutkha/ panmasala }\end{array}$ & 9 & $\begin{array}{l}\text { Maa sharda tobacco, Dasanpura, } \\
\text { Hobli, Banglore }\end{array}$ & $5 /-$ \\
\hline 4 & Hans chhap & Tobacco & 15 & $\begin{array}{l}\text { Murali lal Harish chanderjaiswal, } \\
\text { Preet vihar, Delhi }\end{array}$ & $5 /-$ \\
\hline 5 & Miraj & Tobacco & 10 & Miraj products, Nathdwara, Rajasthan & $5 /-$ \\
\hline 6 & Chaini/Khaini & Tobacco and lime stone paste & 0.36 & $\begin{array}{l}\text { Harsh international industries, } \\
\text { Haryana }\end{array}$ & $15 /-$ \\
\hline 7 & Jora or Blue bull & Tobacco & & & $4 /-$ \\
\hline 8 & Cool lip & Filter tabbaq & 3.6 & $\begin{array}{l}\text { Tej ram dharam paul, Maur Mandi, } \\
\text { Punjab }\end{array}$ & 9/- \\
\hline 9 & Meenaji mawa & Tobacco & & $\begin{array}{l}\text { Meena Jarda Udyog Pvt Ltd, } \\
\text { Bhubaneswar }\end{array}$ & $5 /-$ \\
\hline 10 & Hindustan khaini & $\begin{array}{l}\text { Tobacco, lime, added flavors, and spices } \\
\text { Not a gutkha or panmasala }\end{array}$ & & Peeyush company, Mohanpur, India & $10 /-$ \\
\hline 11 & Badshaha & $\begin{array}{l}\text { Manufactured tobacco } \\
\text { Not a gutkha/ panmasala/ supari }\end{array}$ & & $\begin{array}{l}\text { Damodar Jagannath Malpani fast track } \\
\text { Pvt Ltd, Korahubli, Tumkur }\end{array}$ & $5 /-$ \\
\hline 12 & Zara snus & $\begin{array}{l}\text { Tobacco, lime water, menthol, oil, spices } \\
\text { and added flavors }\end{array}$ & 3.6 & India & $10 /-$ \\
\hline 13 & $\begin{array}{l}\text { Gokul zarda patti } \\
\text { (mawa) }\end{array}$ & $\begin{array}{l}\text { Tobacco, betel nut, natural, synthetic } \\
\text { flavours lime, aromatic spices, sounf } \\
\text { and menthol }\end{array}$ & & $\begin{array}{l}\text { Maa Bhairabi product, Behrampur, } \\
\text { India }\end{array}$ & $4 /-$ \\
\hline 14 & SVS madras snuff & & & $\begin{array}{l}\text { Sri Vijaya Lakshmi snuff products, } \\
\text { Radha Krishna Nagar, Chennai }\end{array}$ & $4 /-$ \\
\hline 15 & Kamath & $100 \%$ unmanufactured tobacco & 8 & $\begin{array}{l}\text { New kamath tobacco Pvt Ltd, PO Mira, } \\
\text { Thane District }\end{array}$ & $4 /-$ \\
\hline 16 & Mangalam gold & Tobacco & 15 & $\begin{array}{l}\text { HN packaging and marketing, } \\
\text { Allawalpur Mohammdabad, } \\
\text { Farrukhabad }\end{array}$ & $10 /-$ \\
\hline
\end{tabular}




\section{Table 2. Gutkha brands available in Andhra Pradesh, India}

No Gutkha brands

1 Khaleja

2 Rebel

$3 \quad$ K909 gutkha

$4 \quad$ Pan parag power

5 Premium nazar gutkha
Effective ingredients

Tobacco company

Price (Rs)

Saffron blended

Saffron blended

Betel nut, catechu, tobacco, menthol, permitted spices, and flavors

Saffron blended gutkha

Betel nut, catechu, tobacco, lime, permitted spices, and flavors
$4 /-$

$4 /-$

Gopalji ketaki khara masala, Berhampur 3/-

Shiva shakti products, Kathmandu, Nepal 4/-

Shrisiddeshwar fragrance Pvt Ltd,

Sitapura, Jaipur, Rajasthan

These tobacco products did not show the COTPA ACT 2003 on the sachets.

Table 3. Panmasala product brands available in Andhra Pradesh, India

\begin{tabular}{|c|c|c|c|c|c|}
\hline No & $\begin{array}{l}\text { Panmasala } \\
\text { brands }\end{array}$ & Effective ingredients & $\begin{array}{c}\text { Net } \\
\text { weight } \\
\text { (g) }\end{array}$ & Tobacco company & $\begin{array}{l}\text { Price } \\
\text { (Rs) }\end{array}$ \\
\hline \multirow[t]{2}{*}{1} & Vimal & $\begin{array}{l}\text { Betel nut, catechu, lime, saffron, spices, } \\
\text { and flavors }\end{array}$ & & VSN Products, Tumkur, Karnataka & $5 /-$ \\
\hline & V-1 Scented & Tobacco & & VS Products, Karnataka & $2 /-$ \\
\hline \multirow[t]{2}{*}{2} & RMD & $\begin{array}{l}\text { Betel nut, catechu, lime, menthol, } \\
\text { cardamom, and permitted flavors }\end{array}$ & & $\begin{array}{l}\text { Dhariwal industries Pvt Ltd, Singasandra } \\
\text { village, Hosur road, Bangalore }\end{array}$ & $7 /-$ \\
\hline & M scented & Tobacco & & $\begin{array}{l}\text { Dhariwal industries Pvt Ltd, Singasandra } \\
\text { village, Hosur road, Bangalore }\end{array}$ & $4 /-$ \\
\hline \multirow[t]{2}{*}{3} & Rajnigandha & No tobacco and no nicotine added flavors & 1.7 & $\begin{array}{l}\text { Dharampal Satyapal (Ltd), } \\
\text { Bhamunimaiden, } \\
\text { Guwahati (Assam) }\end{array}$ & $6 /-$ \\
\hline & Baba 120 & Premium chewing tobacco & 0.45 & $\begin{array}{l}\text { Dharampal premchand (Ltd), sector- } 60 \text {, } \\
\text { Noida }\end{array}$ & $5 /-$ \\
\hline \multirow[t]{2}{*}{4} & Hira & $\begin{array}{l}\text { Blend of kesar and elachi flavors } \\
0 \% \text { tobacco and } 0 \% \text { nicotine }\end{array}$ & & Hira enterprises, Yernal road, Nipani & $1 /-$ \\
\hline & Royale-717 & Tobacco & & Hira enterprises, Yernal road, Nipani & $1 /-$ \\
\hline \multirow[t]{2}{*}{5} & Shanti Strong & $\begin{array}{l}\text { Betelnut, catechu, lime, cardamom seed, } \\
\text { permitted spices, and added flavors }\end{array}$ & & $\begin{array}{l}\text { S.M. Perfumers (P) Ltd, Bommasandra Indl } \\
\text { area, Hosur road, Bangalore }\end{array}$ & $4 /-$ \\
\hline & $\begin{array}{l}\text { SNT } 1000 \text { Zarda } \\
\text { Scented }\end{array}$ & Tobacco & & $\begin{array}{l}\text { S.M. Perfumers (P) Ltd, Bommasandra Indl } \\
\text { area, Hosur road, Bangalore }\end{array}$ & $2 /-$ \\
\hline \multirow[t]{2}{*}{6} & Star 555 & $\begin{array}{l}\text { Betel nut, catechu, tobacco, lime, menthol, } \\
\text { cardamom, and flavors }\end{array}$ & & $\begin{array}{l}\text { Ghodawat industries Pvt Ltd, Hubali, } \\
\text { Dharwad, (Karnataka) }\end{array}$ & $5 /-$ \\
\hline & Star111 & Tobacco & & $\begin{array}{l}\text { Ghodawat Foods international industries } \\
\text { Pvt Ltd, Hubali, Dharwad, (Karnataka) }\end{array}$ & $2 /-$ \\
\hline \multirow[t]{2}{*}{7} & $\begin{array}{l}\text { Parag } 9000 \\
\text { panmasala }\end{array}$ & $\begin{array}{l}\text { Betel nut, catechu, tobacco, lime, menthol, } \\
\text { cardamom, natural, and artificial flavors }\end{array}$ & 1.8 & Panparag India Limited, Allur, Bengaluru & $3 /-$ \\
\hline & $\begin{array}{l}\text { Parag } 9000 \\
\text { tobacco }\end{array}$ & Tobacco & 2.5 & Panparag India Limited, Allur, Bengaluru & $1 /-$ \\
\hline \multirow[t]{2}{*}{8} & $\begin{array}{l}\text { Goa } 1000 \\
\text { panmasala }\end{array}$ & $\begin{array}{l}\text { Betel nuts, catechu, cardamom, lime, } \\
\text { saffron, and flavors }\end{array}$ & & Global technology \& trademarks Ltd, & $2 /-$ \\
\hline & Goa 1000 zarda & Blended chewing tobacco & & $\begin{array}{l}\text { Geluvu food products, vishweshwarapura, } \\
\text { tumkur road, Nelamangla, Banglore }\end{array}$ & $1 /-$ \\
\hline \multirow[t]{2}{*}{9} & $\begin{array}{l}\text { Super gem } \\
\text { panmasala }\end{array}$ & $\begin{array}{l}\text { Betel nut, catechu, cardamom, lime, } \\
\text { menthol, saffron and added flavors }\end{array}$ & & $\begin{array}{l}\text { Thrishul arecanut granules, Siddu } \\
\text { packaging Pvt Ltd }\end{array}$ & $4 /-$ \\
\hline & $\begin{array}{l}\text { Super gem } \\
\text { tobacco }\end{array}$ & Tobacco & & & $1 /-$ \\
\hline
\end{tabular}


Table 3. Continued

\begin{tabular}{|c|c|c|c|c|c|}
\hline No & $\begin{array}{l}\text { Panmasala } \\
\text { brands }\end{array}$ & Effective ingredients & $\begin{array}{l}\text { Net } \\
\text { weight } \\
\text { (g) }\end{array}$ & Tobacco company & $\begin{array}{l}\text { Price } \\
\text { (Rs) }\end{array}$ \\
\hline \multirow[t]{2}{*}{10} & Rajshree & $\begin{array}{l}\text { Betel nut, catechu, cardamom, lime, } \\
\text { menthol, natural and artificial flavors }\end{array}$ & & Kaypan Fragrance Pvt Ltd, Ghaziabad & $4 /-$ \\
\hline & $\begin{array}{l}\text { Kp black label } \\
\text { premium }\end{array}$ & Tobacco & 0.5 & Kaypan Fragrance Pvt Ltd, Ghaziabad & $1 /-$ \\
\hline \multirow[t]{2}{*}{11} & Panparag & $\begin{array}{l}\text { Betel nut, catechu, cardamom, lime, } \\
\text { menthol, natural and artificial flavors } \\
0 \% \text { tobacco and } 0 \% \text { nicotine }\end{array}$ & 3 & $\begin{array}{l}\text { Ruchi flavors LLP, Dashrath Dist, Vadodara. } \\
\text { Panparag India Ltd, Kanpur }\end{array}$ & $4 /-$ \\
\hline & $\mathrm{PP}$ & Chewing tobacco & & $\begin{array}{l}\text { Ruchi flavors LLP, Dashrath Dist, Vadodara. } \\
\text { Panparag India Ltd, Kanpur }\end{array}$ & $2 /-$ \\
\hline \multirow[t]{2}{*}{12} & RR 24 carat & $\begin{array}{l}\text { Betel nut, catechu, cardamom, lime, } \\
\text { menthol, natural and artificial flavors } \\
0 \% \text { tobacco and } 0 \% \text { nicotine }\end{array}$ & & $\begin{array}{l}\text { Unique tobacco products, Bandlaguda, } \\
\text { Post Keshavgiri, Hyderabad }\end{array}$ & $4 /-$ \\
\hline & RR 24 gold & Tobacco & & Everyday products, Bidar, Karnataka & $2 /-$ \\
\hline \multirow[t]{2}{*}{13} & $\mathrm{RR}$ & $\begin{array}{l}\text { No tobacco and no nicotine Betel nut, } \\
\text { catechu, lime, cardamom seeds, and added } \\
\text { flavors }\end{array}$ & 3 & $\begin{array}{l}\text { Unique tobacco products, Bandlaguda, } \\
\text { Post Keshavgiri, Hyderabad }\end{array}$ & $4 /-$ \\
\hline & RR gold & Tobacco & & $\begin{array}{l}\text { Everyday products, Kothari industrial area, } \\
\text { Bidar, Karnataka }\end{array}$ & $2 /-$ \\
\hline \multirow[t]{2}{*}{14} & Sagar & $\begin{array}{l}\text { No tobacco and no nicotine Betel nut } \\
\text { catechu, lime, cardamom, menthol, natural } \\
\text { and artificial flavors }\end{array}$ & & R.K. products, Hyderabad & $4 /-$ \\
\hline & SR-1 & Scented tobacco & & R.K. products, Bidar, Karnataka & $2 /-$ \\
\hline 15 & $\begin{array}{l}\text { Panbahar- } \\
\text { The heritage } \\
\text { panmasala }\end{array}$ & No nicotine and no tobacco added flavors & 2.5 & Ashok \& Co. Panbahar Ltd, Delhi & $5 /-$ \\
\hline 16 & $\begin{array}{l}\text { Aashiqui } \\
\text { supreme saffron } \\
\text { blended }\end{array}$ & $\begin{array}{l}0 \% \text { tobacco and } 0 \% \text { nicotine } \\
\text { Betel nut, catechu, cardamom, lime, } \\
\text { menthol, permitted spices, rose and } \\
\text { kewara flavor }\end{array}$ & & $\begin{array}{l}\text { Mahalakshmi Devi flavors, Pvt Ltd, New } \\
\text { Delhi }\end{array}$ & $5 /-$ \\
\hline \multirow[t]{2}{*}{17} & $\begin{array}{l}\text { Tansen blues- } \\
\text { Flavoured } \\
\text { panmasala }\end{array}$ & $\begin{array}{l}\text { No tobacco and no nicotine } \\
\text { Natural flavouring substances }\end{array}$ & 3 & $\begin{array}{l}\text { Trimurthi Fragrances Pvt Ltd, Patparganj, } \\
\text { Delhi }\end{array}$ & $4 /-$ \\
\hline & Premium zarda & Tobacco & 0.3 & $\begin{array}{l}\text { Trimurthi Fragrances Pvt Ltd, Patparganj, } \\
\text { Delhi }\end{array}$ & $2 /-$ \\
\hline \multirow[t]{2}{*}{18} & $\begin{array}{l}\text { Raj niwas- } \\
\text { Flavoured } \\
\text { panmasala }\end{array}$ & $\begin{array}{l}\text { No tobacco and no nicotine } \\
\text { Betel nut, catechu, cardamom, lime, } \\
\text { menthol, natural, and artificial flavours }\end{array}$ & & $\begin{array}{l}\text { Vardh Paper Products Pvt Ltd, Swarn Park } \\
\text { Indl Area, Mundka, Delhi }\end{array}$ & $4 /-$ \\
\hline & $\begin{array}{l}\text { Maha pasand } \\
\text { zafrani zarda }\end{array}$ & Tobacco & & $\begin{array}{l}\text { Focus township private limited, Swarn } \\
\text { Park Indl Area, Mundka, Delhi }\end{array}$ & $2 /-$ \\
\hline
\end{tabular}

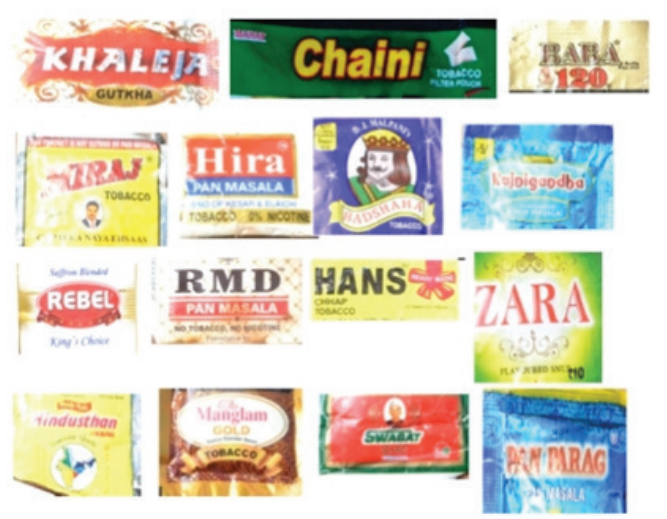

Figure 1. Images of various smokeless tobacco products
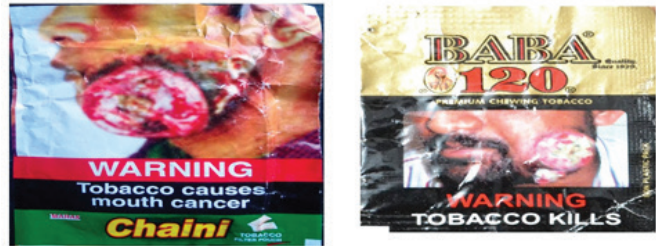

Figure 2. Graphic warning labels on tobacco products, India
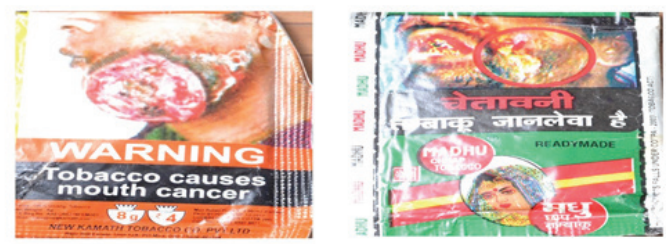
Table 4. Pictorial health warning labels on smokeless tobacco products

\begin{tabular}{|c|c|c|}
\hline No & Product name & Warning label \\
\hline 1 & Premium nazar gutkha & Tobacco kills \\
\hline 2 & Khaini & Tobacco causes mouth cancer \\
\hline 3 & Hindustan khaini & $\begin{array}{l}\text { Chewing of tobacco is injurious } \\
\text { to health and tobacco kills }\end{array}$ \\
\hline 4 & Panmasala & $\begin{array}{l}\text { Chewing of panmasala is/may } \\
\text { be injurious to health }\end{array}$ \\
\hline 5 & Zara snus & $\begin{array}{l}\text { Tobacco is injurious to health, } \\
\text { tobacco causes cancer }\end{array}$ \\
\hline 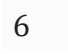 & SVS madras snuff & Tobacco causes mouth cancer \\
\hline
\end{tabular}

tobacco products available (including khaleja and rebel brands of gutkha, chaini brand of khaini, rajnigandha, hira, RMD, and panparag panmasala brands, baba 120 chewing tobacco, miraj, badshaha, hans chhap, zara snus, hindhusthan, manglam gold tobacco, swagat tobacco brands) in the Indian tobacco market.

Most of the sampled smokeless tobacco products have the common pictorial health warning labels: 'Chewing of panmasala is injurious to health; Tobacco causes mouth cancer; Tobacco kills; Chewing of tobacco is injurious to health; and Tobacco is injurious to health' (Table 4).

\section{DISCUSSION}

Our observational study identified numerous sub-brands, ingredients, and warning labels across multiple types of smokeless tobacco products in India, providing a glimpse of a smokeless tobacco market.

The reported smokeless tobacco constituents might contribute to the development of adverse health effects among consumers. Gutkha, panmasala, khaini and tobacco products are the multiple forms of smokeless tobacco that are locally made and consumed throughout India. The common factors of sex, age, ethnic origin, and socioeconomic status showed variation in the pattern of consumption of $\mathrm{SLT}^{20}$. The smokeless tobacco industry is actively engaged in the production of larger number of SLT products, which are addictive nicotine containing products ${ }^{21}$. Nicotine itself and tobacco-specific N-nitrosamines are actively involved in the formation of reactive oxygen species (ROS) in the mouth ${ }^{22,23}$.

Increasing product labelling as a means of increasing public awareness holds significant policy implications, including sustained investment in evidence-based mass media campaigns as part of a comprehensive tobacco control strategy ${ }^{24}$. This should be taken further in light of the evidence that the enactment of smoke-free legislation has been associated with an increased consumption of smokeless tobacco among adolescent males ${ }^{25}$.

The taxes on SLT products remain low compared to smoking cigarette products in Bangladesh and the amendment made in Tobacco Control Law in 2013 requires graphic health warnings to cover $50 \%$ of SLT packaging, a ban on advertisement of SLT products, and restriction of sales to minors $^{26}$. The increased use of smokeless tobacco products is directly proportional to cessation of smoking. The increasing prices of SLT products, which have doubled, may indirectly discourage people from using SLT products ${ }^{27}$. A similar increase in tobacco consumption is occurring globally, with a concomitant increase in tobacco-related deaths and diseases ${ }^{28}$.

\section{Limitations}

This pilot study has a number of limitations due to its specific geographical representation and cross-sectional pack study design. Further research is needed with a broader study area to be able to obtain generalisable results.

\section{CONCLUSIONS}

Smokeless tobacco represents an addictive product in need of specific regulation. The Indian government has enforced tobacco control laws like COTPA ACT 2003 and introduced pictorial health warning labels on packets of SLT products, which are important to protecting public health. As identified by their list of reported ingredients, use of smokeless tobacco products pose a significant health risk, hence the continuation and further strengthening of health warning labels on smokeless tobacco products should be encouraged.

\section{REFERENCES}

1. World Health Organization . WHO Report on the Global Tobacco Epidemic. Geneva, Switzerland: World Health Organization; 2008. https://apps.who.int/iris/bitstream/ handle/10665/43818/9789241596282_eng.pdf;jsessionid= D55FCE242A5BE538925D2FE7E3FB1A87? sequence=1. Accessed March 27, 2019.

2. The NCD Alliance. Tobacco: A major risk factor for noncommunicable diseases. Geneva, Switzerland: The NCD Alliance; 2011. https://ncdalliance.org/sites/default/files/rfiles/NCDA_ Tobacco_and_Health.pdf. Accessed March 27, 2019.

3. National Institutes of Health. Smokeless Tobacco Fact Sheets. In: 3rd International Conference on Smokeless Tobacco, Advancing Science and Protecting Public Health, September 22-25, 2002. http://cancercontrol.Cancer.gov/TCRB/ stfactsheet_combined1023-02.pdf. Assessed July 4, 2008.

4. Stepanov I, Jensen J, Hatsukami D, Hecht SS. New and traditional smokeless tobacco: comparison of toxicant and carcinogen levels. Nicotine Tob Res. 2008;10(12):1773-1782. doi:10.1080/14622200802443544

5. National Cancer Institute, Centers for Disease Control and Prevention. Smokeless tobacco and public health: A global perspective. Bethesda, MD: U.S. Department of Health and Human Services, Centers for Disease Control and Prevention and National Institutes of Health, National Cancer Institute; 2014. https://cancercontrol.cancer.gov/brp/tcrb/globalperspective/SmokelessTobaccoAndPublicHealth.pdf. Accessed March 27, 2019. 
6. Mutti-Packer S, Reid JL, Thrasher JF, Romere D, Fonga GT, Gupta PC, et al. The role of negative affect and message credibility in perceived effectiveness of smokeless tobacco health warning labels in Navi Mumbai, India and Dhaka, Bangladesh: A moderated-mediation analysis. Addict Behav. 2017;73:22-29. doi:10.1016/j.addbeh.2017.04.002

7. Federal Trade Commission. Federal Trade Commission Smokeless tobacco report for 2014. https://www.ftc.gov/ system/files/documents/reports/federal-trade-commissioncigarette-report-2014-federal-trade-commission-smokelesstobacco-report/ftc_cigarette_report_2014.pdf. Accessed March 27, 2019.

8. Elias J, Hendlin Y, Chaffee BW, Ling PM. Don't throw smokeless tobacco users under the bus. Addict Behav. 2017;77:289-290. doi:10.1016/j.addbeh.2017.04.007

9. Food and Drug Administration. Tobacco product standard for N-Nitrosonornicotine level in finished smokeless tobacco products. White Oak, MA: Food and Drug Administration; 2017. https://www.fda.gov/media/102728/download. Accessed March 27, 2019.

10. Cullen JW, Blot W, Henningfield J, Boyd G, Mecklenburg $\mathrm{R}$, Massey MM. Health consequences of using smokeless tobacco: summary of the Advisory Committee's report to the Surgeon General. Public Health Rep. 1986;101(4):355-373. PMID:3090602.

11. World Health Organization, International Agency for Research on Cancer. IARC Monographs on the Evaluation of Carcinogenic Risks to Humans. In: Smokeless Tobacco and Some Tobacco-specific N-Nitrosamines, Vol. 89. Lyon, France: International Agency for Research on Cancer; 2007. https:// monographs.iarc.fr/wp-content/uploads/2018/06/mono89. pdf. Accessed March 27, 2019.

12.Gupta PC, Ray CS. Smokeless tobacco and health in India and South Asia. Respirology. 2003;8(4):419-431. doi:10.1046/j.1440-1843.2003.00507.x

13. Joshi MS, Verma Y, Gautam AK, Shivgotra VK, Parmar G, Kumar $\mathrm{S}$. Assessment of genetic damage among chewers of mixture containing mainly areca nut and tobacco. Asia Pac J Public Health. 2011;23(6):852-860. doi:10.1177/1010539511419838

14. European Commission. Health Effects of Smokeless Tobacco Products. Brussels, Belgium: Scientific Committee on Emerging and Newly Identified Health Risks; 2008. https:// ec.europa.eu/health/ph_risk/committees/04_scenihr/docs / scenihr_o_013.pdf. Accessed March 27, 2019.

15. Government of India. The Cigarettes and Other Tobacco Products (Prohibition of Advertisement and Regulation of Trade and Commerce, Production, Supply and Distribution) Act no. 34, 2003. New Delhi, India: Legislative Department, Ministry of Law and Justice, Government of India; 2003.

\section{CONFLICTS OF INTEREST}

The authors have completed and submitted the ICMJE Form for Disclosure of Potential Conflicts of Interest and none was reported.
http://legislative.gov.in/sites/default/files/A2003-34.pdf. Accessed March 27, 2019.

16. Adedigba MA, Aransiola J, Arobieke RI, Adewoled 0, Oyelamie T. Between traditions and health: beliefs and perceptions of health effects of smokeless tobacco among selected users in Nigeria. Subt Use Misuse. 2017;53(4):565-573. doi:10.1080/10826084.2017.1349796

17. Agaku IT, Singh T, Rolle IV, Ayo-Yusuf OA. Exposure and response to current text-only smokeless tobacco health warnings among smokeless tobacco users aged $\geq 18$ years, United States, 2012-2013. Prev Med. 2016;87:200-206. doi:10.1016/j.ypmed.2016.02.014

18. Klein EG, Queensberry AJ, Shoben AB, Cooper S, Ferketich $\mathrm{AK}$, Berman M, et al. Health warning labels for smokeless tobacco: The impact of graphic images on attention, recall, and craving. Nicotine Tob Res. 2017;19(10):1172-1177. doi:10.1093/ntr/ntx021

19. Hammond D. Health warning messages on tobacco products: a review. Tob Control. 2011;20(5):327-337. doi:10.1136/tc.2010.037630

20. Boffetta P, Hecht S, Gray N, Gupta P, Straif K. Smokeless tobacco and cancer. Lancet Oncol. 2008;9(7):667-675. doi:10.1016/s1470-2045(08)70173-6

21. Benowitz NL. Nicotine Addiction. N Engl J Med. 2010;362(24):2295-2303. doi:10.1056/nejmra0809890

22. Konstantinou E, Fotopoulou F, Drosos A, et al. Tobaccospecific nitrosamines: A literature review. Food Chem Toxicol. 2018;118:198-203. doi:10.1016/j.fct.2018.05.008

23. Niaz K, Maqbool F, Khan F, Bahadar H, Ismail Hassan F, Abdollahi M. Smokeless tobacco (paan and gutkha) consumption, prevalence, and contribution to oral cancer. Epidemiol Health. 2017;39:e2017009. doi:10.4178/epih.e2017009

24. Murukutla N, Yan H, Wang S, Negi NS, Kotov A, Mullin S, Goodchild M. Cost effectiveness of a smokeless tobacco control media campaign in India. Tob Control. 2018;27(5):547-551. doi:10.1136/tobaccocontrol-2016-053564

25. Hawkins SS, Bach N, Baum CF. Impact of tobacco control policies on adolescent smokeless tobacco and cigar use: a difference-in-differences approach. BMC Public Health. 2018;18(1):154. doi:10.1186/s12889-018-5063-z

26. Huque R, Zaman MM, Huq SM, Sinha DN. Smokeless tobacco and public health in Bangladesh. Indian J Public Health. 2017;61(5):18-24. doi:10.4103/ijph.ijph_233_17

27. Kostova D, Dave D. Smokeless tobacco use in India: Role of prices and advertising. Soc Sci Med. 2015;138:82-90. doi:10.1016/j.socscimed.2015.05.036

28. Hammond SK. Global patterns of nicotine and tobacco consumption. Handb Exp Pharmacol. 2009;192:3-28. doi:10.1007/978-3-540-69248-5_1

PROVENANCE AND PEER REVIEW

Not commissioned; externally peer reviewed. 